\title{
Possibilities of Preoperative Medical Models Made by 3D Printing or Additive Manufacturing
}

\begin{abstract}
Mika Salmi
School of Engineering, Department of Mechanical Engineering, Aalto University, Otakaari 4, 02150 Espoo, Finland

Correspondence should be addressed to Mika Salmi; mika.salmi@aalto.fi

Received 8 March 2016; Revised 23 May 2016; Accepted 7 June 2016

Academic Editor: Hengyong Yu

Copyright ( 2016 Mika Salmi. This is an open access article distributed under the Creative Commons Attribution License, which permits unrestricted use, distribution, and reproduction in any medium, provided the original work is properly cited.

Most of the 3D printing applications of preoperative models have been focused on dental and craniomaxillofacial area. The purpose of this paper is to demonstrate the possibilities in other application areas and give examples of the current possibilities. The approach was to communicate with the surgeons with different fields about their needs related preoperative models and try to produce preoperative models that satisfy those needs. Ten different kinds of examples of possibilities were selected to be shown in this paper and aspects related imaging, 3D model reconstruction, 3D modeling, and 3D printing were presented. Examples were heart, ankle, backbone, knee, and pelvis with different processes and materials. Software types required were Osirix, 3Data Expert, and Rhinoceros. Different 3D printing processes were binder jetting and material extrusion. This paper presents a wide range of possibilities related to 3D printing of preoperative models. Surgeons should be aware of the new possibilities and in most cases help from mechanical engineering side is needed.
\end{abstract}

\section{Introduction}

$3 \mathrm{D}$ printing is a process where material is added usually layer by layer as opposite to traditional manufacturing methods. In medical field, 3D printing has huge potential since every patient is unique and customization with $3 \mathrm{D}$ printing required only modifying the 3D model of the product [1].

Medical applications of 3D printing (additive manufacturing) can be categorized into five different groups: (1) medical models, (2) medical aids, orthoses, splints, and prostheses, (3) tools, instruments, and parts for medical devices, (4) inert implants, and (5) biomanufacturing [2]. Similar older application based classifications can be also found by different researchers [3-6]. Hopkinson et al. [3] used terms presurgery rapid manufacturing, orthodontics, drug delivery devices, limb prosthesis, and in vivo devices. Otherwise, Gibson et al. [4] called them surgical and diagnostic aids, prosthetics, and organ printing. In 2009 Mäkitie et al. classified in preoperative planning, surgical training, and teaching, inert implants, surgical instruments, and special equipment associated with the operations, postoperative guides, long-term supports and aids, and artificial tissue. In 2010 Tuomi et al. divided applications to models for preoperative planning, education, and training, inert implants, tools, instruments, and parts for medical devices, medical aids, supportive guides, splints and prostheses, and biomanufacturing. Examples of 3D printing applications include dental applications such as bite splints [7], hard and soft oral applications [8,9] and trachea scaffolds [10], and surgical instruments made by 3D printing [11].

Scientific research has highly focused on implants and biomanufacturing but recently more and more medical preoperative models are made by using $3 \mathrm{D}$ printing for patient treatment. There are multiple cases related to craniomaxillofacial and dental surgery [12-14] and even lots of researches related to accuracy of the medical models made by $3 \mathrm{D}$ printing $[15,16]$. Also guidelines for medical imaging related to making medical models by 3D printing can be found [17], where one major parameter is layer thickness of imaging.

The accuracy of binder jetting has been found varying from $0.69 \%$ to $0.38 \%$ [18] when comparing $3 \mathrm{D}$ printed model to $3 \mathrm{D}$ model of the patient and $3.14 \%$ to $2.67 \%[19,20]$ when comparing 3D printed model to dry bone structure. The accuracy of material extrusion has been found varying from $0.22 \%$ to $1.11 \%[21,22]$ comparing $3 \mathrm{D}$ printed model to $3 \mathrm{D}$ model of the patient. Most measurements are done manually using calipers or with coordinate measuring machine. 
Some reports related to vascular anatomy are found [23] and even some commercial service related this. Still the potential of $3 \mathrm{D}$ printing in preoperative models in other areas has not been discovered and scientific literature is missing. The purpose of this research is to demonstrate possibilities of medical models with $3 \mathrm{D}$ printing in the areas that are so well know.

\section{Materials and Methods}

The process starts from medical imaging. For bony structures and for contrast agents computed tomography is current best solution. The imaging produces medical images in DICOM (Digital Imaging and Communications in Medicine) format and accordingly the image layers 3D model from imaged subject can be reconstructed. This 3D model usually requires removing unwanted geometry and errors before $3 \mathrm{D}$ printing. If the bony structures are not well connected to each other additional support structures are needed to hold the physical model together and these can be 3D designed before manufacturing.

2.1. Heart Model. Heart operation requires well-known anatomy of the heart. Normally hearts consist of similar structures; there are only small size and shape variations. In the cases where there are deformations or heart has been previously operated on there is a need for more accurate anatomic examination. Presented case patient has previous deformations and operations so surgeons feel that preoperative model would help them to plan the surgery beforehand and achieve better results. The heart was imaged with computed tomography, using contrast agent to separate heart from surrounding tissues. Layer thickness in the imaging was $600 \mu \mathrm{m}$. 3D model was reconstructed using Osirix 5.7 (open source) with 130 Hounsfield (HU) value. For other model preparation such as repairing and hollowing 3Data Expert 10.2.1 (DeskArtes Oy) was selected as software. First the different shells were separated from each other using verified shell and repair command. In the same function also gaps thinner than $0.17 \mathrm{~mm}$ were stitched; fill all gaps after that, and remove tiny shell less than $0.01 \%$ of total size. The automatic repairing was performed three times and after that errors left were repaired manually one triangle per time. When the model was repaired enough it was hollowed using offset command with $2 \mathrm{~mm}$ offset. After offsetting model was again automatically and manually repaired. The model was separated into two parts using split command. Two different kinds of 3D model from heart were made, hollow one with 2 pieces and solid one. Both models are shown in Figure 1.

Two models of heart were $3 \mathrm{D}$ printed, one from inner structure with material extrusion process and one with outer structure with binder jetting process. In material extrusion Uprint SE Plus printer (Stratasys Ltd.) was used with layer thickness of $0.254 \mathrm{~mm}$ form ABS plus material. In binder jetting Zprinter 450 (3D Systems, Inc.) was the printing device with layer thickness of $0.1 \mathrm{~mm}$ and ZP151 as material. The hollow one was that which surgeons see more beneficiary since you can see inside, but also solid one gave good view about the anatomy. Biggest problem was that since heart is always pumping blood it moves during imaging and it might be that contrast agent does not flow to each desired location at the same time.

2.2. Ankle Models. Deformation or trauma in the ankle is challenging since there is moving joint in it. It might be hard to understand how different parts of ankle move in the ankle and especially this is hard when the anatomy is abnormal. For imaging ankles, computed tomography was used with layer thickness $625 \mu \mathrm{m}$. For repairing, hollowing, coloring, and other model preparation 3Data Expert 10.1 (DeskArtes Oy) was selected as software and Rhinoceros 4.0 was used to create geometry that connects different bones. The model was first verified and repaired with parameters stich gaps thinner than $0.17 \mathrm{~mm}$; fill all gaps after that and remove tiny shell less than $0.01 \%$ of total size. The automatic repairing was performed two times and couple of errors were repaired manually. For repaired model line with dots was created in Rhinoceros and then solid pipe was created around it. Then the models were solidified to one shell using Boolean operations in 3Data Expert. Two ankles were segmented with $100 \mathrm{HU}$ for both models using Osirix 5.7 (open source). One model was 3D printed with binder jetting method using Zprinter 450 (3D Systems, Inc.) in monochrome and the other with same 3D printer in color mode. For both material was ZP151 and photos of both are shown in Figure 2.

2.3. Models of Backbones. In scoliosis backbone is more in form of $\mathrm{S}$ or $\mathrm{C}$ than straight. In early phases, it can be treated with external support that supports backbone to grow straighter. Since deformation can be in every direction and no common form exists, every patient requires individual treatment. Planning of the treatment is quite hard with only $2 \mathrm{D}$ computed tomography slices or virtual model in computer screen. For 3D printing of backbones computed tomography with layer thickness $625 \mu \mathrm{m}$ was selected. HU value for the first one was $200 \mathrm{HU}$ and for the second one $225 \mathrm{HU}$ when using Osirix 5.7 (open source) software. For repairing, hollowing, coloring, and other model preparation 3 Data Expert 10.1 was used. The models were first verified and repaired with parameters stich gaps thinner than $0.15 \mathrm{~mm}$; fill all gaps after that and remove tiny shell less than $0.01 \%$ of total size. The automatic repairing was performed four times and couple of errors were repaired manually. Rhinoceros 4.0 was used adding supporting tube geometry to connect different vertebras together. Then the models were solidified to one shell using Boolean operations in 3Data Expert.

Since the first one was quite big binder jetting process and VX1000 (voxeljet AG) 3D printer was selected with PMMA as a material, for the second one binder jetting with Zprinter 450 (3D Systems, Inc.) was selected with ZP151 material in full color mode. Scoliosis backbone with binder jetting without and with colors is shown in Figure 3.

2.4. Knee Models. Similar to ankle knee is challenging to operate. There are different bones moving against each other with cartridge between them. From $2 \mathrm{D}$ slice images it is hard to figure out the true $3 \mathrm{D}$ shape of the bones. $3 \mathrm{D}$ printing 

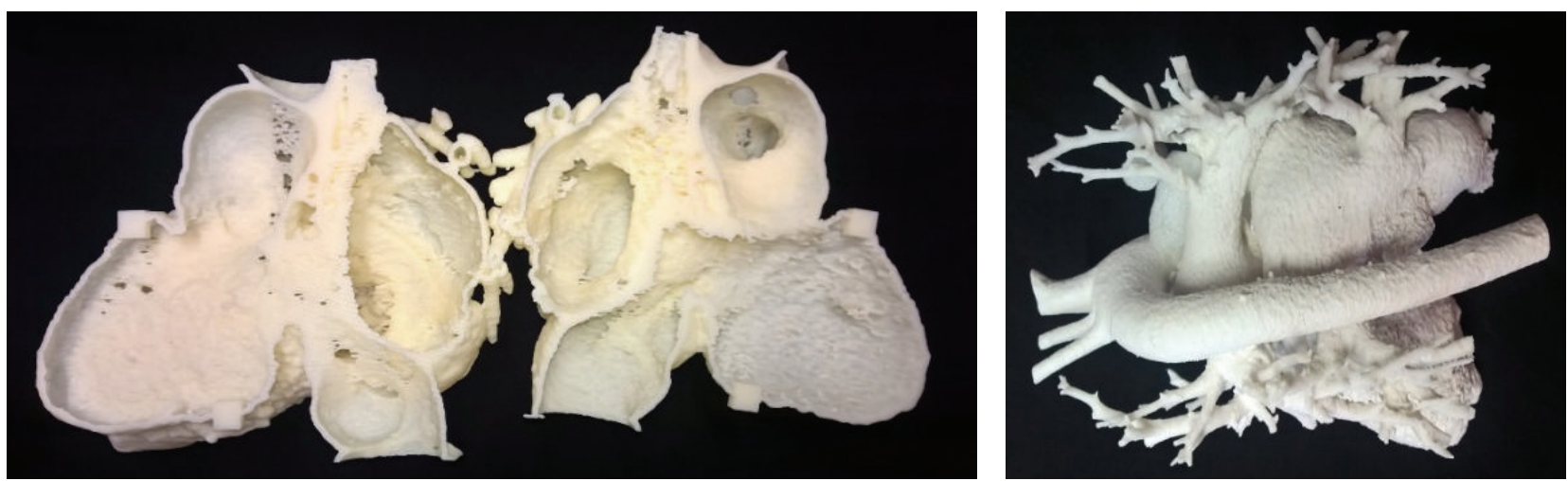

FIGURE 1: Hollow heart made by material extrusion and solid heart made by binder jetting.
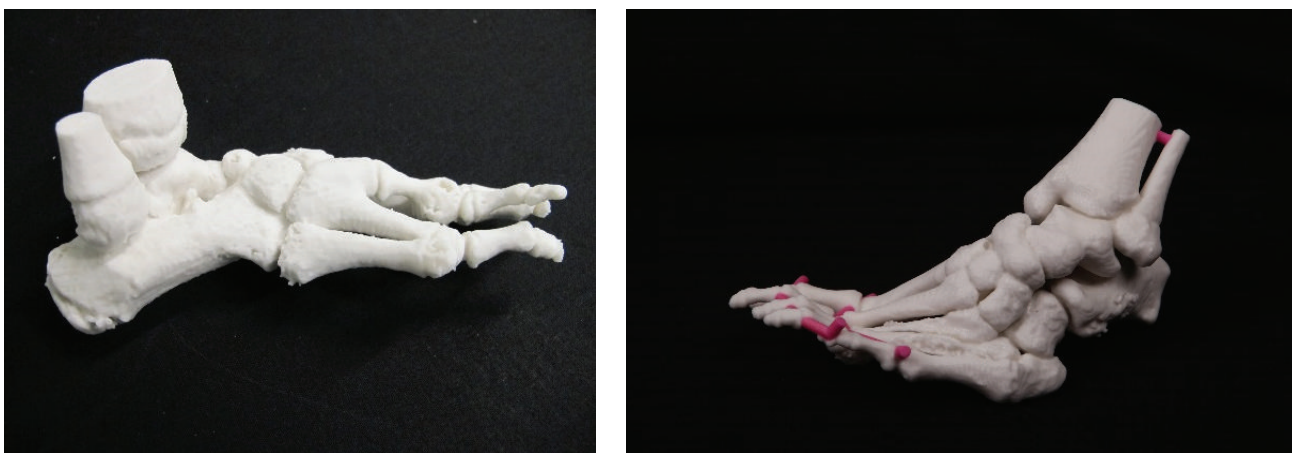

FIGURE 2: Preoperative model of ankle made by binder jetting with monochrome and with colors.
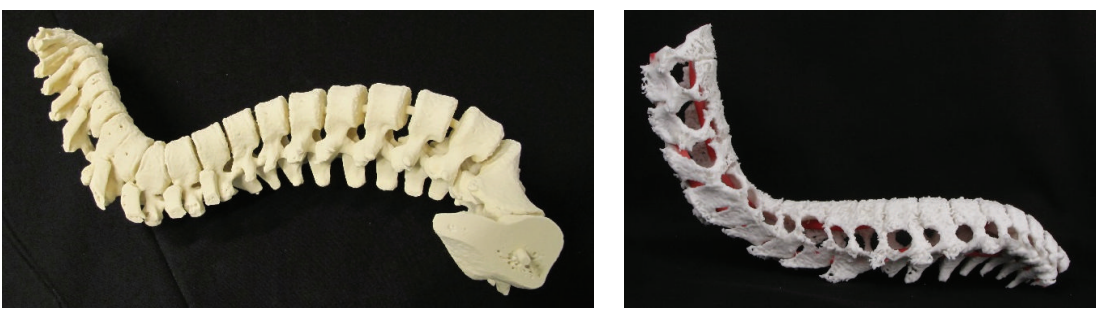

FIGURE 3: Scoliosis backbone with binder jetting without and with colors.

allows seeing replicas of the bones in natural size and touching them. It is also possible to simulate moving of the joint, as imaging computed tomography was used with layer thickness $625 \mu \mathrm{m}$. Both models were segmented using Osirix 5.7 (open source) software with $150 \mathrm{HU}$. The models were first verified and repaired using 3Data Expert 10.1 with parameters stich gaps thinner than $0.2 \mathrm{~mm}$; fill all gaps after that and remove tiny shell less than $0.01 \%$ of total size. The automatic repairing was performed one time and couple of errors were repaired manually. Extra shells were removed before printing.

Binder jetting with Zprinter 450 (3D Systems, Inc.) was selected as a process. The material used was ZP151. Both preoperative knee models were $3 \mathrm{D}$ printed in monochrome mode and in Figure 4 physical model of first one and virtual model of second one are shown.
2.5. Model of Pelvis. Malposition of pelvis affects whole body through backbone and legs. Traumas in this are common with older people related to fall or slip. Sometimes children may have deformation related to pelvis and usually this is related example to scoliosis. For pelvis preoperative models computed tomography with layer thickness $625 \mu \mathrm{m}$ was selected. For segmentation $250 \mathrm{HU}$ was selected for the first one and $300 \mathrm{HU}$ for the second one. Models were repaired, colored, and prepared using 3Data Expert 10.1 (DeskArtes Oy) which was also used to generate support geometry to hold physical model together. First the shells were separated using verified shell and extra shells removed. Repair command was run two times and selected parameters were stich gaps thinner than $0.17 \mathrm{~mm}$; fill all gaps after stitching and remove tiny shell less than $0.01 \%$ of total size. After that errors left 

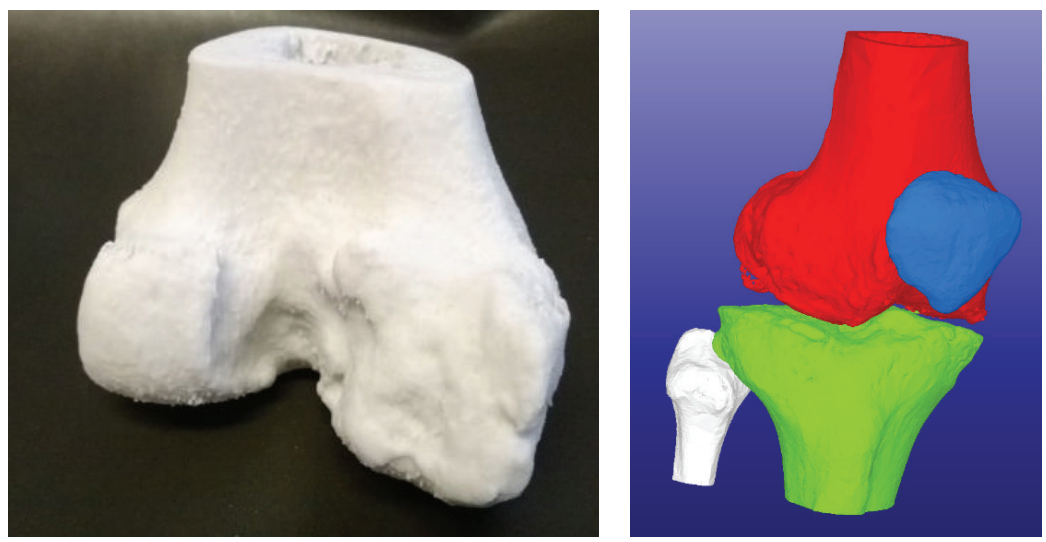

FIgURE 4: Physical and virtual preoperative model of knee.
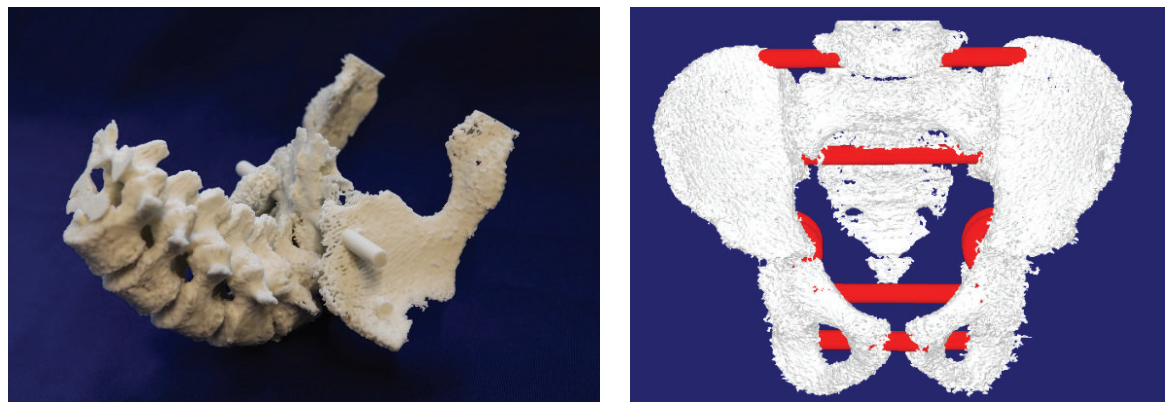

FIGURE 5: Physical and virtual preoperative model of pelvis.

were repaired manually triangle by triangle. After repairing, support geometries were created creating cylinders to the $3 \mathrm{D}$ model and moving cylinders to desired locations. Multiple join operation was used to connect shell together.

Both were 3D printed using binder jetting method with Zprinter 450 (3D Systems, Inc.). The material was ZP151. Photo of the first model and screen capture of virtual model are shown in Figure 5.

2.6. Accuracy of the Models. All models were visually inspected after manufacturing. Two models were selected for more accurate measurements: hollow heart made by material extrusion and preoperative model of knee made by binder jetting. In the heart model inner structure of the other half and in the knee joint surface were 3D measured using ATOS Core 3D (GOM mbH, Germany). The measurements were compared against 3D model used for 3D printing with GOM Inspect V7.5 SR2 (GOM mbH, Germany).

\section{Results and Discussion}

Preoperative models are mostly needed cases where anatomy of the patient varies from normal. This occurs in deformations and with children since their body is still growing and developing and in traumas. Examples 3D printed preoperative models were created from heart, ankle, backbone, knee, and pelvis with different processes and materials. In each case understanding of anatomy was better compared to looking only at 2D slice images. The surgeons estimated that preoperative models helped them to perform surgery. Also better planning reduces the average time used for surgery.

Medical imaging with layers always loses data. When 3D model is created from medical images geometry between layers is always mathematically calculated estimation. The smaller the layer thickness in imaging the better and more accurate the $3 \mathrm{D}$ model created from it. With patients radiation dose leads to the fact that layer thickness cannot be decreased more. In 3D printing selected layer thickness was the smallest one available in the selected printers. The thickness is smaller than layer thickness in imaging. Layer thickness of $3 \mathrm{D}$ printing generates dimensional errors to the physical model but imaging can cause more errors. Also if layer thickness of $3 \mathrm{D}$ printing is too high stair step effect between layers can be seen and it affects the accuracy.

In the heart model made by material extrusion overall accuracy was approximately $\pm 1.5 \mathrm{~mm}$ when comparing $3 \mathrm{D}$ printed model to virtual 3D model. Maximum errors were approximately $\pm 3.0 \mathrm{~mm}$ at thin walls, sharp corners, and small holes. Also the surface of the 3D model and the $3 \mathrm{D}$ print was rough because of imaging soft tissue. In the knee model made by binder jetting overall accuracy was approximately $\pm 0.75 \mathrm{~mm}$ when comparing $3 \mathrm{D}$ printed model to virtual $3 \mathrm{D}$ model. Maximum errors were approximately $\pm 2.5 \mathrm{~mm}$ at sharp corners and small holes. One reason for errors in small holes might be postprocessing where $3 \mathrm{D}$ printed part is dipped in cyanoacrylate and that can accumulate in the holes. 

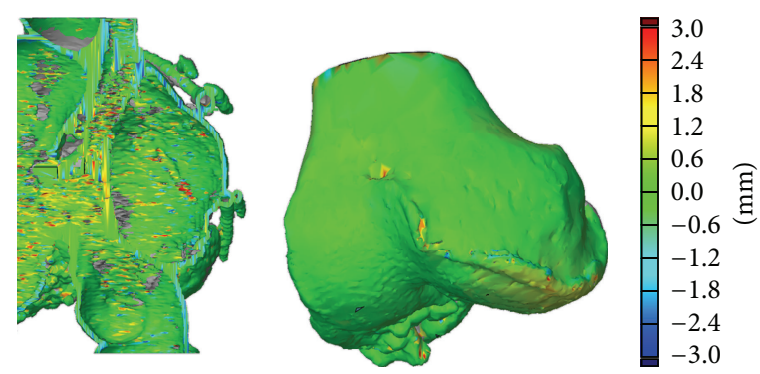

Figure 6: Result of 3D measurement for hollow heart model and for knee model. Scale $\pm 3.0 \mathrm{~mm}$.

The measurement results for both heart model and knee model are shown in Figure 6. Bone structures are more accurate in medical imaging than soft tissue structures and therefore produce better results in 3D printing. Binder jetting was found more accurate in medical models than material extrusion.

Currently preoperative models from bone applications can be easily done if the imaging quality and layer thickness are sufficient. One notice about image quality is that some hospitals remove some parts of images when archiving these for saving storage space. Therefore, images should be taken directly from imaging workspace not from archives. Next applications would be soft tissue and organ applications where more processing of medical images is needed. One possibility is to use contrast agent to increase difference in $\mathrm{HU}$ values between organ and tissue next to it.

Ones that would require development are models made with material jetting process and multimaterial approach. Adding both hard and soft materials to preoperative medical models it would be possible to mimic bone, ligaments, cartilage, and soft tissue in same model.

\section{Conclusion}

In future, more and more applications will be seen from other areas than dental and craniomaxillofacial ones in 3D printing of preoperative models. Surgeons should be aware of the new possibilities and in most cases help from mechanical engineering side is needed. Communication between surgeons and engineers should be developed further and research effort should be focused on preoperative medical models since the potential has not been reached yet. In future a fresh test subject should be imagined using computed tomography, 3D model created from the images, and the 3D model printed. Tissues of fresh subject should be removed and $3 \mathrm{D}$ bone structures measured. The measurement between original bones and 3D printed copy should be compared.

\section{Competing Interests}

The author declares that there are no competing interests regarding the publication of this paper.

\section{Acknowledgments}

The author would like to thank Helsinki University and Helsinki University Hospital for collaboration in medical applications of additive manufacturing.

\section{References}

[1] M. Salmi, Medical applications of additive manufacturing in surgery and dental care [Ph.D. thesis], Aalto University, 2013.

[2] J. Tuomi, K.-S. Paloheimo, J. Vehviläinen et al., "A novel classification and online platform for planning and documentation of medical applications of additive manufacturing," Surgical Innovation, vol. 21, no. 6, pp. 553-559, 2014.

[3] N. Hopkinson, R. Hague, and P. Dickens, Rapid Manufacturing: An Industrial Revolution for the Digital Age, John Wiley \& Sons, New York, NY, USA, 2006.

[4] I. Gibson, D. W. Rosen, and B. Stucker, Additive Manufacturing Technologies: Rapid Prototyping to Direct Digital Manufacturing, Springer, Berlin, Germany, 2010.

[5] A. Mäkitie, K. S. Paloheimo, R. Björkstrand et al., "Medical applications of rapid prototyping-three-dimensional bodies for planning and implementation of treatment and for tissue replacement," Duodecim; Laaketieteellinen Aikakauskirja, vol. 126, no. 2, pp. 143-151, 2009.

[6] J. Tuomi, K. Paloheimo, R. Björkstrand, M. Salmi, M. Paloheimo, and A. A. Mäkitie, "Medical applications of rapid prototyping-from applications to classification," in Proceedings of the 4th International Conference on Advanced Research in Virtual and Physical Prototyping (VRAP '09), pp. 701-704, Leiria, Portugal, October 2009.

[7] M. Salm, K.-S. Paloheimo, J. Tuomi, T. Ingman, and A. Mäkitie, "A digital process for additive manufacturing of occlusal splints: a clinical pilot study," Journal of the Royal Society Interface, vol. 10, no. 84, Article ID 20130203, 2013.

[8] L. Joffe, "Features section: current products and practice invisalign: early experiences," Journal of Orthodontics, vol. 30, no. 4, pp. 348-352, 2003.

[9] M. Salmi, J. Tuomi, R. Sirkkanen, T. Ingman, and A. Mäkitie, "Rapid tooling method for soft customized removable oral appliances," Open Dentistry Journal, vol. 6, no. 1, pp. 85-89, 2012.

[10] J. W. Chang, S. A. Park, J.-K. Park et al., “Tissue-engineered tracheal reconstruction using three-dimensionally printed artificial tracheal graft: preliminary report," Artificial Organs, vol. 38, no. 6, pp. E95-E105, 2014. 
[11] R. Kontio, R. Björkstrand, M. Salmi et al., "Designing and additive manufacturing a prototype for a novel instrument for mandible fracture reduction," Surgery S, vol. 1, pp. 2161-1076, 2012.

[12] S. Gopakumar, "RP in medicine: a case study in cranial reconstructive surgery," Rapid Prototyping Journal, vol. 10, no. 3, pp. 207-211, 2004.

[13] B. Sanghera, S. Naique, Y. Papaharilaou, and A. Amis, "Preliminary study of rapid prototype medical models," Rapid Prototyping Journal, vol. 7, no. 5, pp. 275-284, 2001.

[14] A. Cohen, A. Laviv, P. Berman, R. Nashef, and J. Abu-Tair, "Mandibular reconstruction using stereolithographic 3-dimensional printing modeling technology," Oral Surgery, Oral Medicine, Oral Pathology, Oral Radiology and Endodontology, vol. 108, no. 5, pp. 661-666, 2009.

[15] P. S.-H. Chang, T. H. Parker, C. W. Patrick Jr., and M. J. Miller, "The accuracy of stereolithography in planning craniofacial bone replacement," The Journal of Craniofacial Surgery, vol. 14, no. 2, pp. 164-170, 2003.

[16] D. N. Silva, M. G. De Oliveira, E. Meurer, M. I. Meurer, J. V. L. da Silva, and A. Santa-Bárbara, "Dimensional error in selective laser sintering and 3D-printing of models for craniomaxillary anatomy reconstruction," Journal of Cranio-Maxillofacial Surgery, vol. 36, no. 8, pp. 443-449, 2008.

[17] R. Bibb, D. Eggbeer, and A. Paterson, Medical Modelling: The Application of Advanced Design and Rapid Prototyping Techniques in Medicine, Woodhead, 2014.

[18] M. Salmi, K.-S. Paloheimo, J. Tuomi, J. Wolff, and A. Mäkitie, "Accuracy of medical models made by additive manufacturing (rapid manufacturing)," Journal of Cranio-Maxillofacial Surgery, vol. 41, no. 7, pp. 603-609, 2013.

[19] D. Ibrahim, T. L. Broilo, C. Heitz et al., "Dimensional error of selective laser sintering, three-dimensional printing and PolyJet $^{\mathrm{TM}}$ models in the reproduction of mandibular anatomy," Journal of Cranio-Maxillofacial Surgery, vol. 37, no. 3, pp. 167173, 2009.

[20] D. N. Silva, M. Gerhardt de Oliveira, E. Meurer, M. I. Meurer, J. V. Lopes da Silva, and A. Santa-Bárbara, "Dimensional error in selective laser sintering and 3D-printing of models for craniomaxillary anatomy reconstruction," Journal of CranioMaxillofacial Surgery, vol. 36, no. 8, pp. 443-449, 2008.

[21] I. El-Katatny, S. H. Masood, and Y. S. Morsi, "Error analysis of FDM fabricated medical replicas," Rapid Prototyping Journal, vol. 16, no. 1, pp. 36-43, 2010.

[22] C. Petropolis and D. Kozan, "Accuracy of medical models made by consumer-grade fused deposition modelling printers/La précision de modèles médicaux produits par des imprimantes pour consommation courante faisant appel à la modélisation par dépôt de fil en fusion," The Canadian Journal of Plastic Surgery, vol. 23, no. 2, pp. 91-94, 2015.

[23] F. Rengier, A. Mehndiratta, H. von Tengg-Kobligk et al., “3D printing based on imaging data: review of medical applications," International Journal of Computer Assisted Radiology and Surgery, vol. 5, no. 4, pp. 335-341, 2010. 


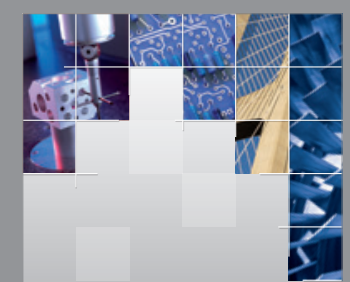

\section{Enfincering}
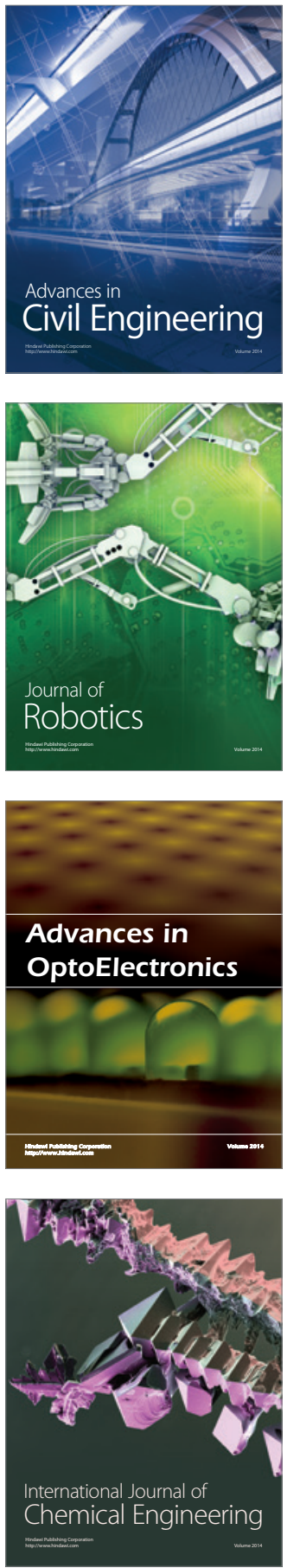

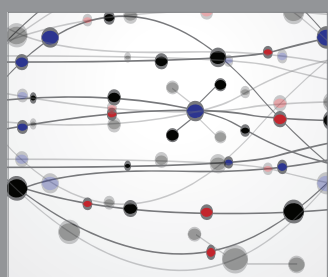

The Scientific World Journal

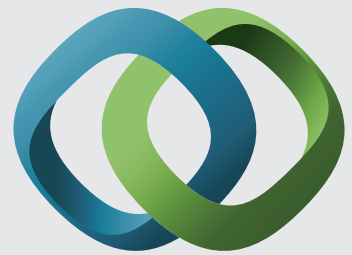

\section{Hindawi}

Submit your manuscripts at

http://www.hindawi.com
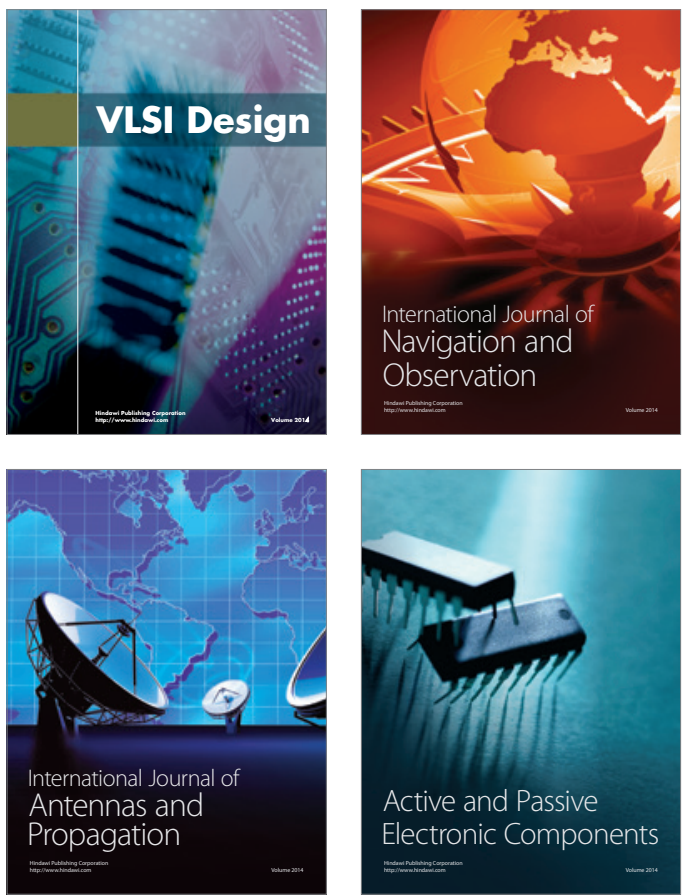
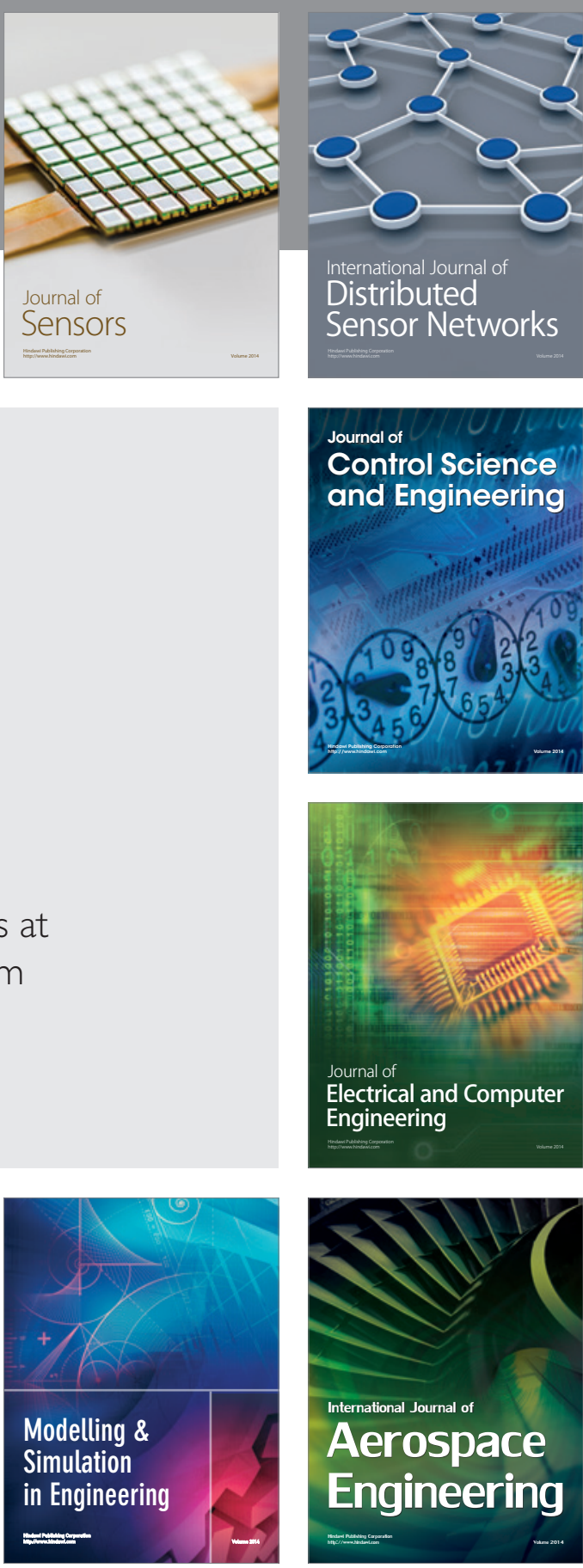

International Journal of

Distributed

Sensor Networks

Journal of

Control Science

and Engineering
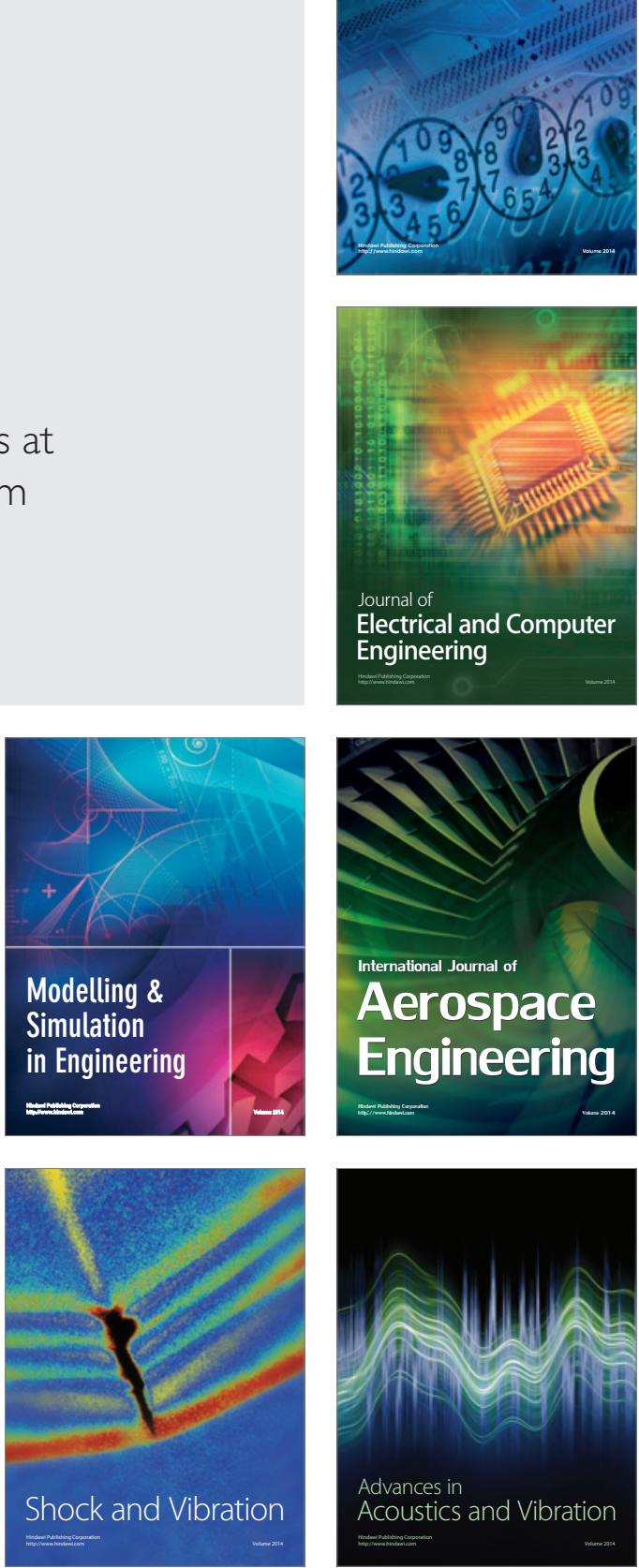